\title{
Finding Your Bagel
}

\author{
Samir S Shah, MD, MSCE
}

Divisions of Hospital Medicine and Infectious Diseases, Cincinnati Children's Hospital Medical Center and the University of Cincinnati College of Medicine, Cincinnati, Ohio.

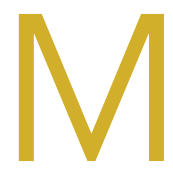

any of us are interested in developing or refining our skillsets. To do so, we need mentorship, which in the still-young field of hospital medicine can sometimes be challenging to obtain.

As a physician-investigator and editor, I commonly encounter young and even mid-career physicians wrestling with how to develop or refine their academic skills, and they're usually pondering the challenges in finding someone in their own division or hospitalist group to help them. When this happens, I talk to them about bagels and cream cheese. I ask them two questions: "What's your cream cheese?" and "Where's your bagel?" Their natural reaction of puzzlement, perhaps mixed with hunger if they haven't yet had breakfast, is similar to the one you've likely just experienced, so let me explain.

In medical school, I had a friend who absolutely loved cream cheese. If it had been socially acceptable, he would have simply walked around scooping cream cheese from a large tub. Had he done that, people would likely have given him funny looks and taken a few steps away. So, instead, my friend found an acceptable solution, which is that he would eat a lot of bagels. And those bagels would be piled high with cream cheese because what he wanted was the cream cheese and the bagel provided a reasonable means by which to get it.

So now I ask you: What's your passion? What is the thing that you want to scoop from the tub (of learning and doing) every day for the rest of your life? That's the cream cheese. Now, all you have to do is to find your bagel, the vehicle that allows you to get there.

Corresponding Author: Samir S Shah, MD, MSCE; Email: Samir.Shah@cchmc.org; Telephone: 513-636-6222; Twitter: @SamirShahMD.

Received: December 14, 2020; Revised: December 19, 2020;

Accepted: December 21, 2020

๑) 2021 Society of Hospital Medicine DOI 10.12788/jhm.3587
Let's see those principles in action. Say that you're a hospitalist who wants to learn how to conduct randomized clinical trials, enhance medication reconciliation, or improve transitions of care. You can read about randomization schemes or improvement cycles but that's clearly not enough. You need someone to help you frame the question, understand how to navigate the system, and avoid potential pitfalls. You need someone with relevant experience and expertise, someone with whom you can discuss nuances such as the trade-offs between different outcome measures or analytic approaches. You need your bagel.

There may not be anyone in your division with such expertise. You may need to branch out to find that bagel. You talk to a few people and they all point you to a cardiologist who runs clinical trials. What other field has such witty study acronyms as MRFIT or MIRACL or PROVE IT? If you're interested in medication reconciliation, they may direct you to a pharmacist who studies medication errors. If you're interested in improving care transitions, they may connect you with a critical care physician with expertise in interhospital transfers. You can meet with these folks to learn about their work. If their personality and mentorship style are a good fit, you can offer to assist in some aspect of their ongoing studies and, in return, ask for mentorship. You may have only a limited interest in the clinical content area, but if there is someone willing to invest their time in teaching, mentoring, and sponsoring you, then you've found your bagel.

Think about what you're hoping to accomplish and keep an open mind to unexpected venues for mentorship and skill development. That bagel may be in your division or department, or it may be somewhere else in your institution, or it may not be in your institution at all but elsewhere regionally or nationally. The sequence is important. What's your cream cheese? Figured it out? Great, now go find that bagel.

Disclosure: The author has no conflicts to disclose. 